\section{Go-ahead for first peanut allergy drug}

The US Food and Drug Administration has approved a first food allergy drug, Aimmune Therapeutics' Palforzia, for children 4-17 years old with peanut allergies. Palforzia (AR101) consists of peanut (Arachis hypogaea) powder capsules to help patients build up tolerance to accidental peanut exposure. Patients start the oral desensitization course with 3-mg daily dose of peanut protein and gradually build up to a 300-mg daily maintenance dose. In a 496-patient pivotal trial, $67 \%$ of Palforzia recipients tolerated a 600-mg peanut protein challenge, after 6 months on maintenance treatment, with only mild allergic reactions. Only $4 \%$ of placebo recipients tolerated this challenge.

Peanut allergy affects around 1 million children in the United States, and accidental exposure can provoke life-threatening anaphylactic shock in some patients, leading Aimmune to predict the drug could exceed $\$ 1$ billion in global annual sales. But others have their doubts. At over $\$ 10,000$ per year, the drug's high cost could deter insurers, and its side effect profile - which mirrors the effects of peanut exposure - could put patients off, say some. While some patients might be tempted by a do-ityourself peanut desensitization program, clinicians caution against it because of the increased need for epinephrine while on treatment. For Palforzia, treatment initiation and dose escalation must take place in a supervised medical setting.

Aimmune's closest competitor is DBV Technologies. The biotech, headquartered in Paris, first filed its transdermal peanut tolerance patch Viaskin Peanut for approval in 2018, but withdrew this submission months later pending more manufacturing and quality control data. It resubmitted the patch in 2019 and anticipates a decision by August. Aimmune and DBV Technologies are also working on other food allergy desensitization products, including products for egg allergy and milk allergy.

\section{Single-cell RNA-seq analysis software providers scramble to offer solutions}

\author{
A raft of tools have sprung up to help biologists work through the single-cell \\ transcriptomic bottleneck, but integration remains elusive.
}

te he market for single-cell analytical technologies is booming: from a value of $\$ 1.83$ billion in 2018 , it could triple by 2025 , according to a report from ResearchAndMarkets.com published last year. The research community's enthusiastic embrace of single-cell RNAseq has already transformed the fortunes of some instrument and kit manufacturers. 10X Genomics CEO Serge Saxonov, in a Q3 earnings call from November 2019, announced that the near-term market for his company's technologies for single-cell analysis could reach $\$ 13$ billion. But this rapid uptake could also create headaches for new users as they learn how to come to grips with the complexities of single-cell data.

For over a decade, researchers have been developing RNA sequencing (RNAseq) techniques to analyze changes in gene expression in individual cells rather than whole tissue samples. This technology is allowing researchers to tackle complex biological systems and phenomena - for example, profiling rare cell types within a tissue, analyzing tumor heterogeneity, or tracing lineages in differentiating cells at single-cell resolution. Achieving such insights requires specialized algorithms that can account for the distinctive artifacts and biases associated with data arising from these experiments. "Single-cell data is much noisier than bulk RNA-seq," says biostatistician Stephanie Hicks, of the Johns Hopkins Bloomberg School of Public Health. computational tools to overcome that noise, as the scale of analysis expands, other challenges will grow too.

The scientific community has eagerly embraced single-cell RNA-seq (scRNA-seq). This interest has given impetus to academics' Although bioinformaticians have devised

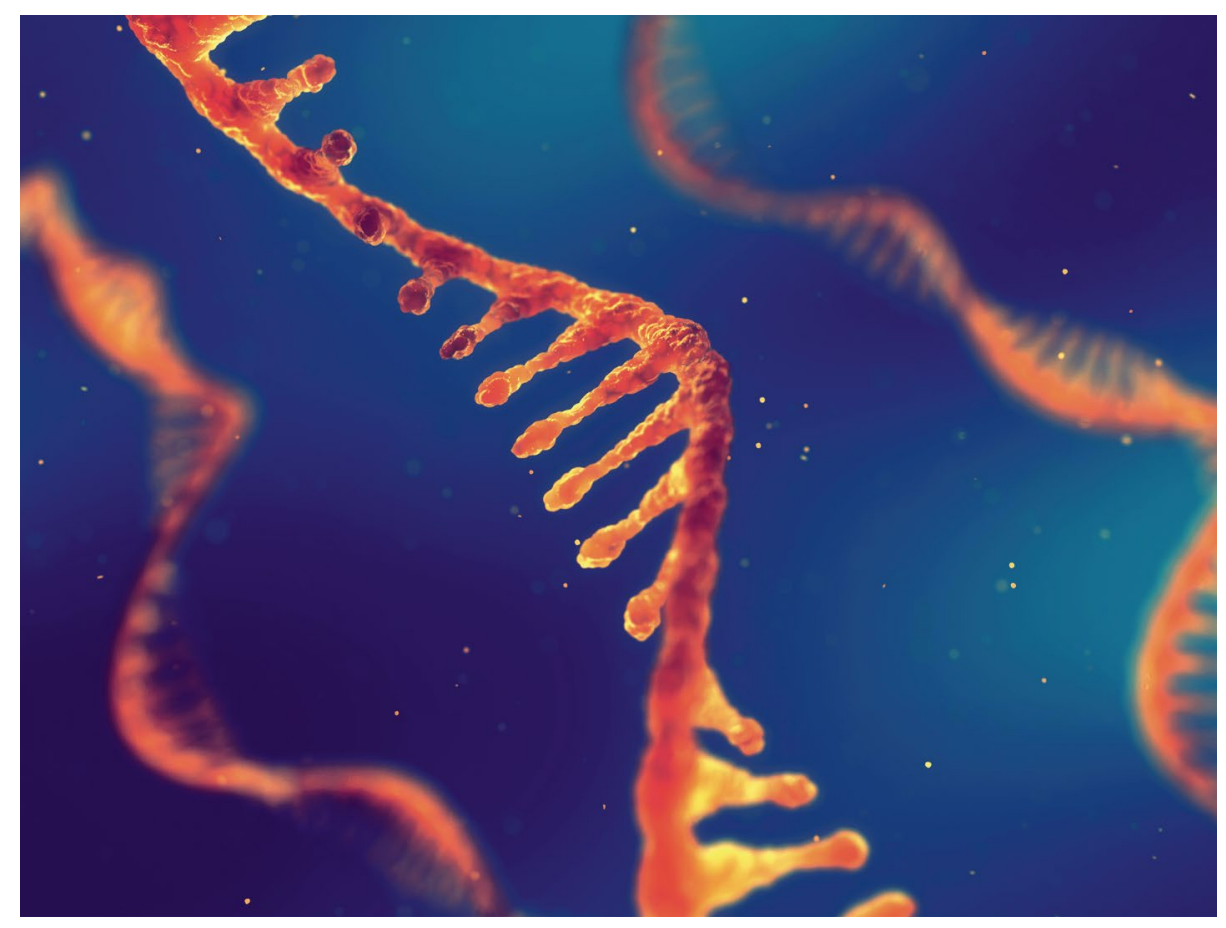

Making sense of single-cell RNA-seq data can be problematic for labs without in-house bioinformatics capabilities. Credit: Nobeastsofierce Science / Alamy Stock Photo 